OMAE2009-79116

\title{
PRELIMINARY INSTABILITY-ANALYSIS OF DEEPWATER RISER WITH FAIRINGS
}

\author{
Mahdi Khorasanchi and Shan Huang \\ Joint Department of Naval Architecture and Marine Engineering \\ Universities of Glasgow and Strathclyde \\ 100 Montrose Street, Glasgow G4 0LZ, UK
}

\begin{abstract}
Instability of deepwater riser with fairings is investigated in this study. Despite the advantages over other devices for suppressing vortex-induced-vibration (VIV), fairings may be susceptible to flutter type instability. A two-body mathematical model is established for the coupled transverse-torsion motion of a top tensioned riser with fairings. The inner part (riser) can only move transversely while the outer part (fairing) has transverse-torsion motion. The effect of the transverse velocity on the angle of attack is taken into account and damping is considered for both degrees of freedom. An eigenvalue analysis is employed to examine the issue of stability. The emphasis is on identifying the critical current speed for a given riser and fairing configuration. The effects of key parameters are investigated and the results indicate that the section hydrodynamic characteristics of the fairings have a significant impact on the instability.
\end{abstract}

\section{INTRODUCTION}

Marine risers are subjected to drag and vortex induced vibration (VIV). The drag is important, particularly for deepwater drilling risers, as it can lead to suspension of operations or even riser disconnection. VIV on the other hand can significantly shorten the riser fatigue life. A number of VIV suppression devices have been proposed and/or used in practice. Among these, helical strake is probably the most prevalent option. The main shortcoming of the helical strake is that it increases the drag force. In contrast, it is possible to reduce the drag and suppress VIV simultaneously by the use of fairings.

Fairings are typically of teardrop geometry, as shown in Figure 1, varying in terms of chord length, nose thickness, span length, tip and tail details. They reduce the vibration by streamlining the flow and weakening the vortices shed aft of the fairing. Compared with the helical strakes, fairings have better performance in VIV suppression (Allen, 2003). They are also more effective than others when located downstream in the wake of upstream risers. Moreover, they reduce the drag as well. But the downside is that fairings may be susceptible to flutter-type instability, as observed in some studies (Lee and Allen, 2005, Meyer et al., 1995, Slocum et al., 2004, Ericsson and Reding, 1980, Ikeda et al., 2003). A key feature observed in the model tests, which is characteristically different from VIV, is that the vibration amplitude increases monotonically with the current velocity.

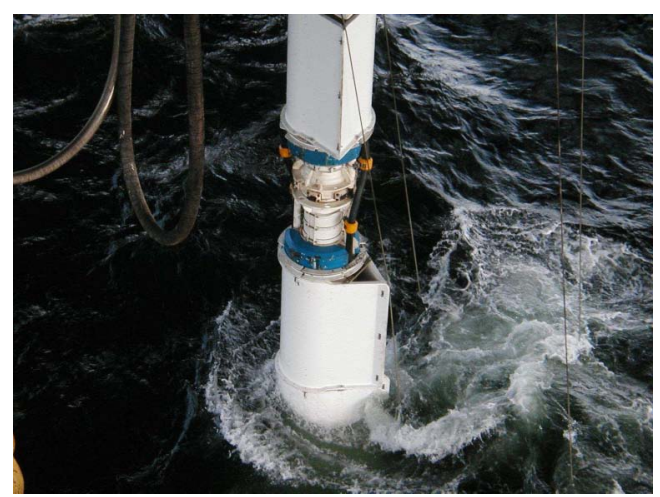

Figure 1 A Typical Installed Riser Fairing.

Slocum et al. (2004) demonstrated that a simple model of aircraft wing flutter can explain the mechanism of riser fairing instability and help to better understand the cause of oscillation. Although connected, riser and fairing are two independent bodies. A single body modelling may be overly simplistic, for 
example, it can not handle the friction between the two bodies which may play an important part to the onset of the instability.

\section{NOMENCLATURE}

$\mathrm{A}_{1}, \mathrm{~A}_{2} \quad$ Area of riser and fairing

c Fairing chord length (tip to tail)

$\mathrm{C}_{\mathrm{D}} \quad$ Drag coefficient measured at centre of rotation

$\mathrm{C}_{\mathrm{L}} \quad$ Lift coefficient measured at centre of rotation

$\mathrm{C}_{\mathrm{M}(\mathrm{cr})} \quad$ Moment coefficient measured at centre of rotation

$\mathrm{C}_{\mathrm{y}}, \mathrm{C}_{\theta} \quad$ Transverse and torsional damping coefficients

$\mathrm{k}_{\mathrm{y}} \quad$ Transverse stiffness

$\mathrm{T}_{1}, \mathrm{~T}_{2} \quad$ Kinetic energy

$\mathrm{U} \quad$ Current velocity

$\mathrm{U}_{\text {rel }} \quad$ Relative current velocity

$\mathrm{U}_{\mathrm{ry}} \quad$ Reduced velocity

V Potential energy

y Transverse motion

$\alpha \quad$ Angle of attack (AoA)

$\theta \quad$ Torsional motion

$\xi, \eta \quad$ Local coordinates

$\rho \quad$ Fluid density

$\rho_{\mathrm{s}} \quad$ Structural density

\section{MATHEMATICAL MODELLING}

Consider a simplified 2D model of a riser and its fairing. The local coordinate system, $(\xi, \eta)$, is fixed on the fairing at its centre of rotation (cr), as shown in Figure 2. Only the transverse motion of the riser, $y$, is considered. The fairing follows the riser's transverse motion, as well as rotates about its centre of rotation with the rotation angle defined as $\theta$. In a fixed global coordinate system $(X, Y)$, the horizontal and vertical velocities of any point on the fairing can be written as,

$\dot{\mathrm{X}}=\eta \dot{\theta}$ and $\dot{\mathrm{Y}}=\dot{\mathrm{y}}-\xi \dot{\theta}$

The kinetic energy of the system is made up of two parts, the energy of solid bodies $\left(\mathrm{T}_{1}\right)$, i.e. riser and fairing, and the energy of fluid motion $\left(\mathrm{T}_{2}\right)$ induced by moving bodies,

$$
\begin{aligned}
\mathrm{T} & =\mathrm{T}_{1}+\mathrm{T}_{2}= \\
& =\frac{1}{2} \int_{\mathrm{A}_{1}} \dot{\mathrm{y}}^{2} \rho_{\mathrm{s}} \mathrm{d} \xi \mathrm{d} \eta+\frac{1}{2} \int_{\mathrm{A}_{2}}\left(\dot{\mathrm{X}}^{2}+\dot{\mathrm{Y}}^{2}\right) \rho_{\mathrm{s}} \mathrm{d} \xi \mathrm{d} \eta \\
& +\frac{1}{2} \int_{\text {fluid }}\left[\left(\dot{\mathrm{y}}_{\mathrm{x}}^{\dot{\mathrm{y}}}+\dot{\theta} \mathrm{u}_{\mathrm{x}}^{\dot{\theta}}\right)^{2}+\left(\dot{\mathrm{y}}_{\mathrm{y}} \dot{\mathrm{y}}+\dot{\theta} \mathrm{u}_{\mathrm{y}}\right)^{2}\right] \rho \mathrm{dA} \\
& =\frac{1}{2} \mathrm{my}^{2}+\frac{1}{2} \mathrm{~J} \dot{\theta}^{2}-\mathrm{S}_{\mathrm{x}} \dot{\mathrm{y}} \dot{\theta}+\frac{1}{2} \mathrm{~m}_{\mathrm{a}} \dot{\mathrm{y}}^{2}+\frac{1}{2} \mathrm{~J}_{\mathrm{a}} \dot{\theta}^{2}-\mathrm{S}_{\mathrm{a}} \dot{\mathrm{y}} \dot{\theta}
\end{aligned}
$$

where

$$
\begin{aligned}
& \mathrm{m}=\int_{\mathrm{A}_{1}+\mathrm{A}_{2}} \rho_{\mathrm{s}} \mathrm{dA} \\
& \mathrm{J}=\int_{\mathrm{A}_{2}}\left(\eta^{2}+\xi^{2}\right) \rho_{\mathrm{s}} \mathrm{dA}
\end{aligned}
$$

$\mathrm{S}_{\mathrm{x}}=\int_{\mathrm{A}_{2}} \xi \rho_{\mathrm{s}} \mathrm{dA}$

$\mathrm{m}_{\mathrm{a}}=\int_{\text {fluid }}\left(\left(\mathrm{u}_{\mathrm{x}}^{\dot{\mathrm{y}}}\right)^{2}+\left(\mathrm{u}_{\mathrm{y}}^{\dot{\mathrm{y}}}\right)^{2}\right) \rho \mathrm{dA}$

$\mathrm{J}_{\mathrm{a}}=\int_{\text {fluid }}\left(\left(\mathrm{u}_{\mathrm{x}}^{\dot{\theta}}\right)^{2}+\left(\mathrm{u}_{\mathrm{y}}^{\dot{\theta}}\right)^{2}\right) \rho \mathrm{dA}$

$\mathrm{S}_{\mathrm{a}}=-\int_{\text {fluid }}\left(\mathrm{u}_{\mathrm{x}}^{\dot{\mathrm{y}}} \mathrm{u}_{\mathrm{x}}^{\dot{\theta}}+\mathrm{u}_{\mathrm{y}}^{\dot{\mathrm{y}}} \mathrm{u}_{\mathrm{y}}^{\dot{\theta}}\right) \rho \mathrm{dA}$

where $\rho_{\mathrm{s}}$ and $\rho$ are structural and fluid densities, respectively. $\mathrm{u}_{\mathrm{x}}^{\dot{y}}$ denotes the fluid particle's velocity in $x$ direction, induced by unit velocity of fairing in $y$ direction.

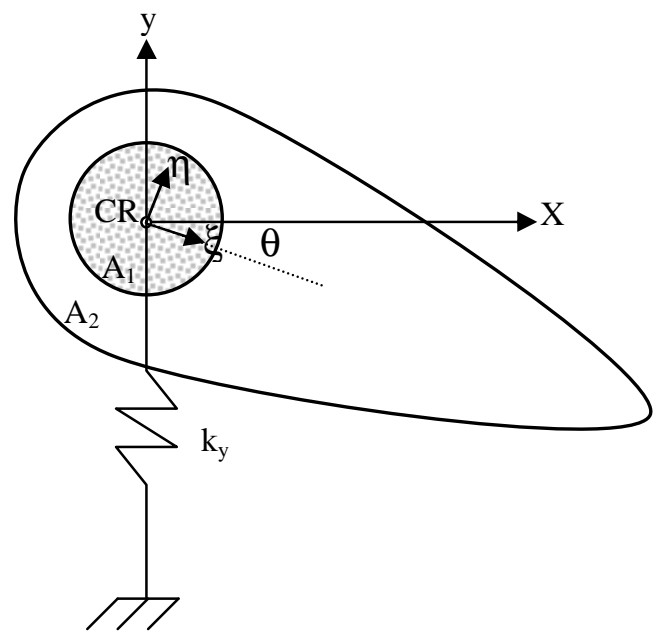

Figure 2 Local and Global Coordinate Systems.

The potential energy (V) is given as,

$\mathrm{V}=\frac{1}{2} \mathrm{k}_{\mathrm{y}} \mathrm{y}^{2}$

Lagrange's equation for dynamics states,

$\mathrm{Q}_{\mathrm{i}}=\frac{\mathrm{d}}{\mathrm{dt}}\left(\frac{\partial}{\partial \dot{\mathrm{q}}_{\mathrm{i}}}(\mathrm{T}-\mathrm{V})\right)-\frac{\partial}{\partial \mathrm{q}_{\mathrm{i}}}(\mathrm{T}-\mathrm{V})$

where $q_{i}$ shows the $i^{\text {th }}$ degree of freedom and $Q_{i}$ is the corresponding external force. The external force includes the hydrodynamic forces and linear damping, $\mathrm{C}_{\mathrm{y}}$ and $\mathrm{C}_{\theta}$, i.e.,

$\mathrm{Q}_{\mathrm{y}}=[\text { Hydrodynamic Force }]_{\mathrm{y}}-\mathrm{C}_{\mathrm{y}} \dot{\mathrm{y}}$

$\mathrm{Q}_{\theta}=[\text { Hydrodynamic Force }]_{\theta}-\mathrm{C}_{\theta} \dot{\theta}$ 
When the current moves with velocity of $U$ in $\mathrm{x}$ direction, if the quasi-steady state is considered, both the transverse and torsional velocities have an effect on the relative flow velocity, $\mathrm{U}_{\text {rel }}$, and change the real angle of attack (Blevins, 2001) as shown in Figure 3,

$\beta=\arctan \left(\frac{\dot{\mathrm{y}}-\mathrm{R} \dot{\theta}}{\mathrm{U}}\right)$

$\alpha=\theta-\beta$

$\mathrm{R}$ shows the position of a reference point for the velocity induced by the angular velocity (Blevins, 2001). If $\mathrm{R}>0$, it means this point is aft of the centre of rotation. of,

Hydrodynamic force for each degree of freedom consists

$[\text { Hydro. force }]_{\mathrm{y}}=(\mathrm{Lift}) \times \cos \beta-($ Drag $) \times \sin \beta=$

$$
\left.\frac{1}{2} \rho \mathrm{U}_{\text {rel }}{ }^{2} \mathrm{c} \mathrm{C}_{\mathrm{L}}\right|_{\alpha} \times \cos \beta-\left.\frac{1}{2} \rho \mathrm{U}_{\text {rel }}{ }^{2} \mathrm{c} \mathrm{C}_{\mathrm{D}}\right|_{\alpha} \times \sin \beta
$$

$[\text { Hydro. force }]_{\theta}=\left.\frac{1}{2} \rho \mathrm{U}_{\mathrm{rel}}{ }^{2} \mathrm{c}^{2} \mathrm{C}_{\mathrm{M}(\mathrm{cr})}\right|_{\alpha}$

where $\mathrm{c}$ is the chord length of the fairing. $\left.\mathrm{C}_{\mathrm{L}}\right|_{\alpha},\left.\mathrm{C}_{\mathrm{D}}\right|_{\alpha}$, and $\left.\mathrm{C}_{\mathrm{M}(\mathrm{cr})}\right|_{\alpha}$ are the lift, drag and moment coefficients at incident angle of $\alpha$, measured at the centre of rotation (cr).

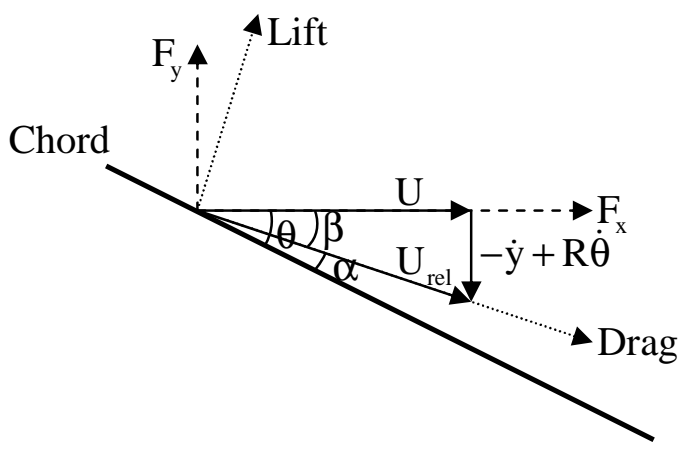

Figure 3 Effective Angle of Attack $\alpha$.

The equations of motion can now be written as

$$
\begin{gathered}
\left(\mathrm{m}+\mathrm{m}_{\mathrm{a}}\right) \ddot{\mathrm{y}}+\mathrm{C}_{\mathrm{y}} \dot{\mathrm{y}}-\left(\mathrm{S}_{\mathrm{x}}+\mathrm{S}_{\mathrm{a}}\right) \ddot{\theta}+\mathrm{k}_{\mathrm{y}} \mathrm{y}= \\
\frac{1}{2} \rho \mathrm{U}_{\mathrm{rel}}{ }^{2} \mathrm{c}\left(\left.\mathrm{C}_{\mathrm{L}}\right|_{\alpha} \cos \beta-\left.\mathrm{C}_{\mathrm{D}}\right|_{\alpha} \sin \beta\right) \\
-\left(\mathrm{S}_{\mathrm{x}}+\mathrm{S}_{\mathrm{a}}\right) \ddot{\mathrm{y}}+\left(\mathrm{J}+\mathrm{J}_{\mathrm{a}}\right) \ddot{\theta}+\mathrm{C}_{\theta} \dot{\theta}=\left.\frac{1}{2} \rho \mathrm{U}_{\text {rel }}{ }^{2} \mathrm{c}^{2} \mathrm{C}_{\mathrm{M}(\mathrm{cr})}\right|_{\alpha}
\end{gathered}
$$

For a small angle of attack, the parameters $\beta,\left.\mathrm{C}_{\mathrm{L}}\right|_{\alpha},\left.\mathrm{C}_{\mathrm{D}}\right|_{\alpha}$ and $\left.\mathrm{C}_{\mathrm{M}(\mathrm{cr})}\right|_{\alpha}$ can be linearised as

$$
\begin{aligned}
& \left.\mathrm{C}_{\mathrm{L}}\right|_{\alpha}=\left.\mathrm{C}_{\mathrm{L}}\right|_{\mathrm{o}}+\left.\alpha \frac{\partial \mathrm{C}_{\mathrm{L}}}{\partial \alpha}\right|_{\mathrm{o}}+\mathrm{O}\left(\alpha^{2}\right) \\
& \left.\mathrm{C}_{\mathrm{D}}\right|_{\alpha}=\left.\mathrm{C}_{\mathrm{D}}\right|_{\mathrm{o}}+\left.\alpha \frac{\partial \mathrm{C}_{\mathrm{D}}}{\partial \alpha}\right|_{\mathrm{o}}+\mathrm{O}\left(\alpha^{2}\right) \\
& \left.\mathrm{C}_{\mathrm{M}(\mathrm{cr})}\right|_{\alpha}=\left.\mathrm{C}_{\mathrm{M}(\mathrm{cr})}\right|_{\mathrm{o}}+\left.\alpha \frac{\partial \mathrm{C}_{\mathrm{M}(\mathrm{cr})}}{\partial \alpha}\right|_{\mathrm{o}}+\mathrm{O}\left(\alpha^{2}\right) \\
& \beta=\arctan \left(\frac{\dot{\mathrm{y}}-\mathrm{R} \dot{\theta}}{\mathrm{U}}\right)=\left(\frac{\dot{\mathrm{y}}-\mathrm{R} \dot{\theta}}{\mathrm{U}}\right)+\mathrm{O}\left(\left(\frac{\dot{\mathrm{y}}-\mathrm{R} \dot{\theta}}{\mathrm{U}}\right)^{2}\right)
\end{aligned}
$$

where $\mathrm{O}\left(\alpha^{2}\right)$ means terms proportional to $\alpha^{2}$ and higher powers of $\alpha$ have been neglected.

By keeping only the linear terms, the governing equations are in the form of,

$$
\begin{aligned}
& \left(\mathrm{m}+\mathrm{m}_{\mathrm{a}}\right) \ddot{\mathrm{y}}+\left(\mathrm{C}_{\mathrm{y}}+\frac{1}{2} \rho \mathrm{Uc}\left(\left.\frac{\partial \mathrm{C}_{\mathrm{L}}}{\partial \alpha}\right|_{\mathrm{o}}+\left.\mathrm{C}_{\mathrm{D}}\right|_{\mathrm{o}}\right)\right) \dot{\mathrm{y}}+\mathrm{k}_{\mathrm{y}} \mathrm{y} \\
& -\left(\mathrm{S}_{\mathrm{x}}+\mathrm{S}_{\mathrm{a}}\right) \ddot{\theta}-\frac{1}{2} \rho \mathrm{RUc}\left(\left.\frac{\partial \mathrm{C}_{\mathrm{L}}}{\partial \alpha}\right|_{\mathrm{o}}+\left.\mathrm{C}_{\mathrm{D}}\right|_{\mathrm{o}}\right) \dot{\theta}-\left(\left.\frac{1}{2} \rho \mathrm{U}^{2} \mathrm{c} \frac{\partial \mathrm{C}_{\mathrm{L}}}{\partial \alpha}\right|_{\mathrm{o}}\right) \theta \\
& =\left.\frac{1}{2} \rho \mathrm{U}^{2} \mathrm{c} \mathrm{C}_{\mathrm{L}}\right|_{\mathrm{o}} \\
& -\left(\mathrm{S}_{\mathrm{x}}+\mathrm{S}_{\mathrm{a}}\right) \ddot{\mathrm{y}}+\left(\left.\frac{1}{2} \rho \mathrm{Uc}^{2} \frac{\partial \mathrm{C}_{\mathrm{M}(\mathrm{cr})}}{\partial \alpha}\right|_{\mathrm{o}}\right) \dot{\mathrm{y}}+\left(\mathrm{J}+\mathrm{J}_{\mathrm{a}}\right) \ddot{\theta} \\
& +\left(\mathrm{C}_{\theta}-\left.\frac{1}{2} \rho R \mathrm{Rc}^{2} \frac{\partial \mathrm{C}_{\mathrm{M}(\mathrm{cr})}}{\partial \alpha}\right|_{\mathrm{o}}\right) \dot{\theta}-\left(\left.\frac{1}{2} \rho \mathrm{U}^{2} \mathrm{c}^{2} \frac{\partial \mathrm{C}_{\mathrm{M}(\mathrm{cr})}}{\partial \alpha}\right|_{\mathrm{o}}\right) \theta \\
& =\left.\frac{1}{2} \rho \mathrm{U}^{2} \mathrm{c}^{2} \mathrm{C}_{\mathrm{M}(\mathrm{cr})}\right|_{\mathrm{o}}
\end{aligned}
$$

By defining the following,

$\hat{\mathrm{Y}}=\dot{\mathrm{y}}$ and $\hat{\Theta}=\dot{\theta}$

Eqs (8) and (9) can be re-written in a matrix form,

$\underline{\underline{\mathrm{A}}} \cdot \underline{\dot{\mathrm{X}}}=\underline{\mathrm{B}} \cdot \underline{\mathrm{X}}$

$$
\underline{X}=\left\{\begin{array}{c}
y \\
\hat{Y} \\
\theta \\
\hat{\Theta}
\end{array}\right\}
$$

The eigen-value equation is

$\operatorname{det}[\underline{\underline{A} \lambda}-\underline{B}]=0$

and the condition for stability is $\operatorname{Real}(\lambda)<0$. 
The last term on the left hand side of Equation (9) can be interpreted as hydrodynamic torsional stiffness. Thus, the natural frequencies of both motions are defined as,

$$
\begin{aligned}
& \omega_{\mathrm{y}}=2 \pi \mathrm{f}_{\mathrm{y}}=\sqrt{\frac{\mathrm{k}_{\mathrm{y}}}{\mathrm{m}+\mathrm{m}_{\mathrm{a}}}} \\
& \omega_{\theta}=2 \pi \mathrm{f}_{\theta}=\sqrt{-\left.\frac{1}{2} \rho \mathrm{U}^{2} \mathrm{c}^{2} \frac{\partial \mathrm{C}_{\mathrm{M}(\mathrm{cr})}}{\partial \alpha}\right|_{\mathrm{o}} /\left(\mathrm{J}+\mathrm{J}_{\mathrm{a}}\right)}
\end{aligned}
$$

To non-dimensionalise the eigen-value equation, the following dimensionless parameters are defined,

$\tilde{\lambda}=\frac{\lambda}{\mathrm{U} / \mathrm{c}}$

$\gamma^{2}=\frac{J+J_{a}}{\left(m+m_{a}\right) \cdot c^{2}}$

$\mathrm{S}_{\mathrm{r}}=\frac{\mathrm{S}_{\mathrm{x}}+\mathrm{S}_{\mathrm{a}}}{\left(\mathrm{m}+\mathrm{m}_{\mathrm{a}}\right) \cdot \mathrm{c}}$

$\mathrm{A}=\frac{\rho \cdot c^{2}}{2\left(\mathrm{~m}+\mathrm{m}_{\mathrm{a}}\right)}$

$\xi_{y}=\frac{C_{y}}{2 \cdot\left(m+m_{a}\right) \cdot \omega_{y}}$

$\xi_{\theta}=\frac{\mathrm{C}_{\theta}}{2 \cdot\left(\mathrm{J}+\mathrm{J}_{\mathrm{a}}\right) \cdot \omega_{\theta}}$

$\mathrm{U}_{\mathrm{ry}}=\frac{\mathrm{U}}{\omega_{\mathrm{y}} \cdot \mathrm{c}}$

$\mathrm{U}_{\mathrm{r} \theta}=\frac{\mathrm{U}}{\omega_{\theta} \cdot \mathrm{c}}=\frac{\gamma}{\sqrt{\left.\mathrm{A} \frac{-\partial \mathrm{C}_{\mathrm{M}(\mathrm{cr})}}{\partial \alpha}\right|_{\mathrm{o}}}}$

$\mathrm{R}_{\mathrm{r}}=\frac{\mathrm{R}}{\mathrm{c}}$

where $\tilde{\lambda}$ is the ratio of the time that takes a flow particle to pass the chord to the period of oscillation; $\gamma$ is the dimensionless form of the radius of gyration about pivot point with respect to chord length; $S_{\mathrm{r}}$ is the dimensionless distance of centre of gravity from pivot point; $\mathrm{A}$ is the inverse of mass ratio; $\xi_{\mathrm{y}}$ and $\xi_{\theta}$ are the damping ratio of transverse and torsional motions respectively; $\mathrm{U}_{\mathrm{ry}}$ and $\mathrm{U}_{\mathrm{r} \theta}$ are reduced velocities; and $\mathrm{R}_{\mathrm{r}}$ is the dimensionless distance of reference point. by,

The non-dimensional eigen-value equation is then given

$\sum_{i=1}^{4} \tilde{c}_{i} \tilde{\lambda}^{i}=0$

where

$$
\begin{aligned}
& \tilde{\mathrm{c}}_{4}=\left[\gamma^{2}-\mathrm{S}_{\mathrm{r}}{ }^{2}\right] \\
& \tilde{\mathrm{c}}_{3}=\left[\left.\mathrm{A}\left(\mathrm{S}_{\mathrm{r}}-\mathrm{R}_{\mathrm{r}}\right) \frac{\partial \mathrm{C}_{\mathrm{M}(\mathrm{cr})}}{\partial \alpha}\right|_{0}\right. \\
& +\mathrm{A}\left(\gamma^{2}-\mathrm{R}_{\mathrm{r}} \mathrm{S}_{\mathrm{r}}\right)\left(\left.\frac{\partial \mathrm{C}_{\mathrm{L}}}{\partial \alpha}\right|_{\mathrm{o}}+\left.\mathrm{C}_{\mathrm{D}}\right|_{\mathrm{o}}\right) \\
& \left.+2 \gamma^{2}\left(\frac{\xi_{\mathrm{y}}}{\mathrm{U}_{\mathrm{ry}}}+\frac{\xi_{\theta}}{\mathrm{U}_{\mathrm{r} \theta}}\right)\right] \\
& \tilde{\mathrm{c}}_{2}=\left[\frac{\gamma^{2}}{\mathrm{U}_{\mathrm{ry}}^{2}}-\left.\mathrm{A}\left(1+2 \mathrm{R}_{\mathrm{r}} \frac{\xi_{\mathrm{y}}}{\mathrm{U}_{\mathrm{ry}}}\right) \frac{\partial \mathrm{C}_{\mathrm{M}(\mathrm{cr})}}{\partial \alpha}\right|_{\mathrm{o}}\right. \\
& +2 \mathrm{~A} \gamma^{2} \frac{\xi_{\theta}}{\mathrm{U}_{\mathrm{r} \theta}}\left(\left.\frac{\partial \mathrm{C}_{\mathrm{L}}}{\partial \alpha}\right|_{\mathrm{o}}+\left.\mathrm{C}_{\mathrm{D}}\right|_{\mathrm{o}}\right) \\
& \left.+4 \gamma^{2} \frac{\xi_{\mathrm{y}}}{\mathrm{U}_{\mathrm{ry}}} \frac{\xi_{\theta}}{\mathrm{U}_{\mathrm{r} \theta}}-\left.\mathrm{AS}_{\mathrm{r}} \frac{\partial \mathrm{C}_{\mathrm{L}}}{\partial \alpha}\right|_{\mathrm{o}}\right] \\
& \tilde{\mathrm{c}}_{1}=\left[-\left.\mathrm{A}\left(2 \frac{\xi_{\mathrm{y}}}{\mathrm{U}_{\mathrm{ry}}}+\frac{\mathrm{R}_{\mathrm{r}}}{\mathrm{U}_{\mathrm{ry}}^{2}}\right) \frac{\partial \mathrm{C}_{\mathrm{M}(\mathrm{cr})}}{\partial \alpha}\right|_{0}\right. \\
& \left.+2 \frac{\gamma^{2}}{\mathrm{U}_{\mathrm{ry}}{ }^{2}} \frac{\xi_{\theta}}{\mathrm{U}_{\mathrm{r} \theta}}-\left.\left.\mathrm{A}^{2} \frac{\partial \mathrm{C}_{\mathrm{M}(\mathrm{cr})}}{\partial \alpha}\right|_{\mathrm{o}} \mathrm{C}_{\mathrm{D}}\right|_{\mathrm{o}}\right] \\
& \tilde{\mathrm{c}}_{0}=\left[-\left.\mathrm{A} \frac{1}{\mathrm{U}_{\mathrm{ry}}^{2}} \frac{\partial \mathrm{C}_{\mathrm{M}(\mathrm{cr})}}{\partial \alpha}\right|_{\mathrm{o}}\right]
\end{aligned}
$$

This equation is solved for $\tilde{\lambda}$ to see if the condition $\operatorname{Real}(\lambda)<0$ is satisfied.

\section{VERIFICATION AND PARAMETRIC STUDY}

A series of rigid cylinder tests was carried out by ExxonMobil at the David Taylor Model Basin (Slocum et al., 2004). One of the tests was on a rigid cylinder with the diameter of $22 \mathrm{~cm}$ and the length of $3.96 \mathrm{~m}$. It was fitted with six independent and identical fairing segments with their profile given in Figure 4. Each fairing segment has a span of $61.2 \mathrm{~cm}$, chord length of $52.6 \mathrm{~cm}$, and thickness of $23.2 \mathrm{~cm}$.

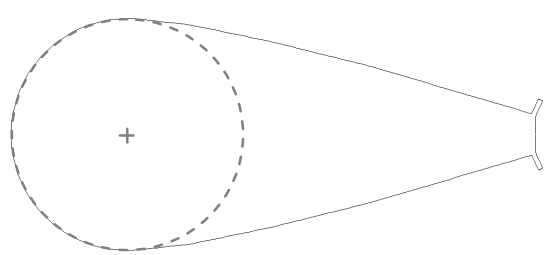

Figure 4 ExxonMobil Fairing on a Rigid Cylinder. 
Based on the data provided in the paper, some of the dimensionless parameters were calculated and given below:

$\mathrm{A}=0.8435$

$\gamma^{2}=0.0792$

$\mathrm{S}_{\mathrm{r}}=0.2016$

$R_{r}$ is a reference length for average effect of angular velocity on real angle of attack (AoA). For flutter of a thin airfoil which rotates about its elastic axis, $R_{r}$ is chosen to give the AoA at a point three-quarters of the chord length back from the leading edge (Fung, 2002). The elastic axis for a typical airfoil is aft of midpoint and close to it. But the thick section of fairing swings about the centre of riser, further up toward the leading edge. Thus, $\mathrm{R}_{\mathrm{r}}$ was selected as 0.40 .

The other group of required data in the model relates to hydrodynamic behaviour of fairing. A CFD analysis was performed on a few typical fairings including this section. The analysis was done at Reynolds number of $5 \times 10^{4}$ at different angles of attack. The velocity contour in the vicinity of the fairing at zero AoA is illustrated in Figure 5.

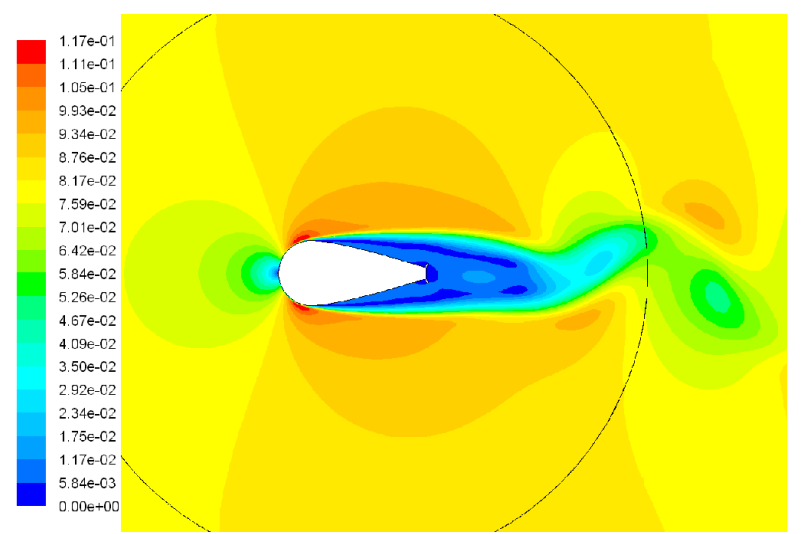

Figure 5 CFD Results - Velocity Distribution at $\operatorname{Re} 5 \times 10^{4}$.

Based upon the above study, the following parameter values are obtained.

$\left.\mathrm{C}_{\mathrm{D}}\right|_{\mathrm{o}}=0.176$

$\partial \mathrm{C}_{\mathrm{L}} /\left.\partial \alpha\right|_{\mathrm{o}}=1.146(1 / \mathrm{rad})$

$\partial \mathrm{C}_{\mathrm{M}(\mathrm{cr})} /\left.\partial \alpha\right|_{\mathrm{o}}=-0.0344(1 / \mathrm{rad})$

With respect to damping, some experimental tests report high level of in-water damping for riser fitted with fairing, e.g. 0.10 to 0.18 (Lee et al., 2004). Very little data on torsional friction damping is available. For the preliminary study here, the following damping ratios are selected.

$$
\begin{aligned}
& \xi_{y}=5 \% \\
& \xi_{\theta}=1 \%
\end{aligned}
$$

The critical reduced velocity, $\mathrm{U}_{\mathrm{cr}}$, at which the real part of solution to equation (17) becomes positive, is obtained numerically by increasing the reduced velocity and solving this equation at each increment. For this case study, the analytical model shows the system becomes unstable at $\mathrm{U}_{\mathrm{cr}}=\mathrm{U}_{\mathrm{ry} \text { (flutter) }}=$ 0.51 , which is in the range of experimental results $(0.42-0.56)$ (Slocum et al., 2004). Through the analysis of eigenvalues, the model also reveals that the transverse mode becomes first unstable in this case. The trajectories of the eigenvalues in the complex plane as the current velocity increases are shown in Figure 6. The four eigenvalues were calculated for a range of reduced velocities, starting from zero with a small increment. The red circles correspond to the highest reduced velocity investigated, where one pair of the eigenvalues crosses the imaginary axis with its real part becoming positive.

The imaginary part of the solution, $\tilde{\lambda}$, relates to the frequency of vibration. According to equations (15) and (16), the imaginary and real parts are multiplied by $\mathrm{U}_{\mathrm{ry}}$ to remove the effect of velocity in the non-dimensionlisation, i.e.,

$$
\tilde{\lambda} \times \mathrm{U}_{\text {ry }}=\frac{\lambda}{\omega_{\mathrm{y}}}
$$

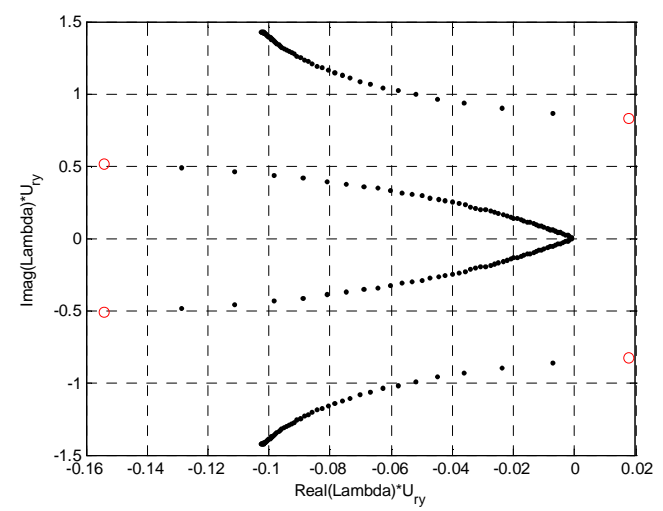

Figure 6 Trajectory of Eigenvalues with Increasing Velocity.

It is seen in Figure 6 that as the current speed increases, the imaginary part of eigenvalues associated with the torsional mode (left branch), increases too. This is in conformity with the fact that higher current velocity generates larger hydrodynamic torsional stiffness and consequently higher frequency of vibration. It should be reminded that the last term in the left- 
hand side of equation (9) can be interpreted as the only hydrodynamic torsional stiffness which is proportional to the current speed squared.

The variation of the critical reduced velocity, $U_{c r}$, against the fairing section hydrodynamic coefficients is demonstrated in Figure 7 to Figure 9. It is seen from these figures that the slopes of the lift and moment curves at $\alpha=0$ play a significant role in the stability of system. On the other hand, the drag force does not present any significant influence on the instability onset condition in this case.

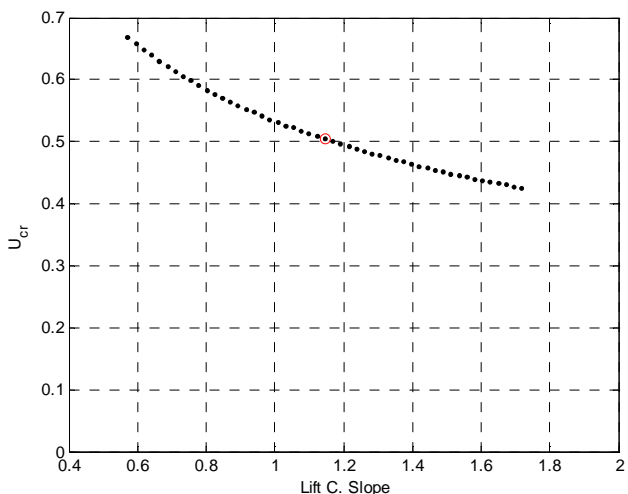

Figure 7 Critical Reduced Velocity versus Lift-Curve Slope at $\alpha=0$.

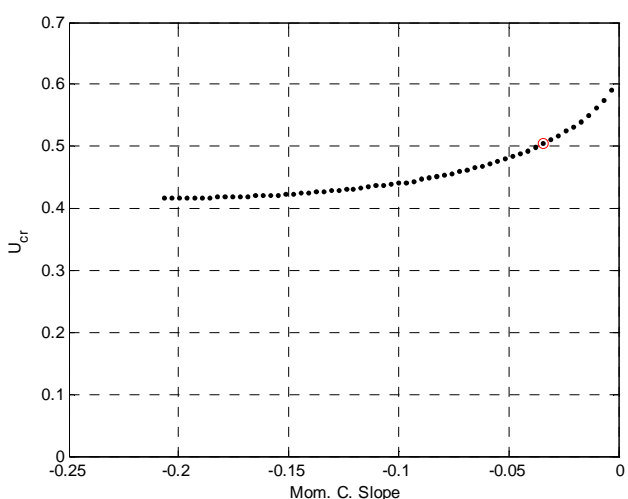

Figure 8 Critical Reduced Velocity versus Moment-Curve Slope at $\alpha=0$.

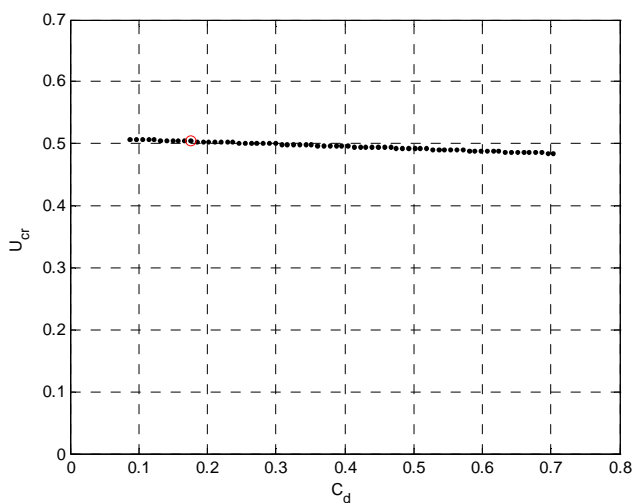

Figure 9 Critical Reduced Velocity versus Drag Coefficient.

\section{CONCLUSION}

The study investigated the dynamic instability of riser fairing and developed a more general analytical model to predict the onset condition of instability. The critical condition, at which the combined system of riser and fairing loses its stability, was formulated as a function of the structural properties of the riser and the hydrodynamic characteristics of the fairing. In the model, the real angle of attack is influenced by both the transverse and angular motions. It also accounted for the effect of damping. Preliminary results of the mathematical model showed a good agreement with test data. It was also concluded that the section hydrodynamic properties of the fairing are critical to the instability onset condition.

\section{REFERENCES}

ALLEN, D. W. (2003) Performance Characteristics of Short Fairings. Offshore Technology Conference (OTC03-15285). Houston, U.S.A.

BLEVINS, R. D. (2001) Flow-Induced Vibrations, Florida, USA, Krieger Publishing Company.

ERICSSON, L. E. \& REDING, J. P. (1980) Potential Hydroelastic Instability of Profiled Underwater Structures. Journal of Hydronautics, 14, 97-104.

FUNG, Y. C. (2002) Theory of Aeroelasticity, New York, USA, Dover Publications.

IKEDA, Y., KATAYAMA, T. \& ENOMOTO, T. (2003) Reduction of Inclination and Vortex-Induced-Oscillation of a Spar Buoy in Rapid Current. 13th International Offshore and Polar Engineering Conference. Honolulu, USA.

LEE, L. \& ALLEN, D. W. (2005) The Dynamic Stability of Short Fairings. Offshore Technology Conference (OTC0517125). Houston, USA. 
LEE, L., ALLEN, D. W., HENNING, D. L. \& MCMULLEN, D. (2004) Damping Characteristics of Fairings for Suppressing Vortex-Induced Vibrations. 23th International Conference on Offshore Mechanics and Arctic Engineering (OMAE04-51209). Vancouver, Canada, American Society of Mechanical Engineers (ASME).

MEYER, P. A., FORT, J. A., SHEKARRIZ, A. \& RUSSELL, D. A. (1995) Hydrodynamic Performance of an Offshore Drilling Riser Fairing. 3rd International Symposium on Performance Enhancement for Marine Applications. Newport, USA.

SLOCUM, S. T., DING, Z. J., FRANK, W. R. \& COX, M. R. (2004) Flutter Instability in Riser Fairings. Offshore Technology Conference (OTC04-16342). Houston, USA. 